Marquette University

e-Publications@Marquette

Biomedical Engineering Faculty Research and

Publications

Biomedical Engineering, Department of

$7-20-2020$

\title{
The Effect of Discrete Visual Perturbations on Balance Control during Gait
}

Lara I. Riem

Brian D. Schmit

Scott A. Beardsley

Follow this and additional works at: https://epublications.marquette.edu/bioengin_fac

Part of the Biomedical Engineering and Bioengineering Commons 
Marquette University

e-Publications@Marquette

\title{
Biomedical Engineering Faculty Research and Publications/College of
} Engineering

This paper is NOT THE PUBLISHED VERSION.

Access the published version via the link in the citation below.

2020 42nd Annual International Conference of the IEEE Engineering in Medicine \& Biology Society (EMBC), (July 20-24, 2020). DOI. This article is (C) The Institute of Electrical and Electronics Engineers and permission has been granted for this version to appear in e-Publications@Marquette. The Institute of Electrical and Electronics Engineers does not grant permission for this article to be further copied/distributed or hosted elsewhere without the express permission from The Institute of Electrical and Electronics Engineers.

\section{The Effect of Discrete Visual Perturbations on Balance Control during Gait}

\author{
Lara I. Riem \\ Marquette University and Medical college of Wisconsin, Milwaukee, WI \\ Brian D. Schmit \\ Marquette University and Medical college of Wisconsin, Milwaukee, WI \\ Scott A. Beardsley \\ Marquette University and Medical college of Wisconsin, Milwaukee, WI
}

\footnotetext{
Abstract:

Immersive virtual reality provides a safe and costeffective approach to administrating balance disruption during ambulation. Previous research has explored the effects of applying continuous perturbations in a virtual environment to challenge balance. This pilot study investigates the ability to disrupt balance with discrete visual perturbations during ambulation in healthy young adults. During the study participants walked on a treadmill within a virtual environment. As they walked the entire visual scene was intermittently shifted to the left or right
} 
1 meter over 1 second. The results demonstrate a significant decrease in step length $(p<; 0.05)$ and change in center of mass excursion ( $p<; 0.05$ ) across participants $(N=13)$. Changes in gait lasted up to three steps after application, suggesting a consistent challenge to dynamic balance control as a result of the discrete visual perturbation. Further, participants did not demonstrate a reduction in response to the discrete visual perturbation with repeated exposure. The results indicate that discrete visual perturbations of a virtual scene can be used to challenge gait and modulate center of mass sway. The use of visual perturbations within a virtual environment to challenge dynamic balance could provide a safer and more affordable avenue for balance rehabilitation by reducing the need for systems that physically perturb balance.

\section{SECTION I. Introduction}

Proper control of balance during ambulation is an important aspect of rehabilitation in elderly[1] and patient populations[2]. Challenges to balance have proven to be an effective rehabilitation regimen, including the application of forces via pullies[3], movement of the support surface[2], or placement of obstacles in the environment[4]. As virtual reality (VR) has become more accessible, studies have shown the effectiveness of VR for rehabilitation in multiple sclerosis[5], stroke[6] and following spinal cord injury [7].

Dynamic balance (i.e., balance during non-stance tasks) can be challenged and improved by providing mechanical perturbations in concert with VR[2]. However, the effectiveness of using VR itself, without the expense of systems that apply physical perturbations, has not been well studied. Recently, Riem et al. [8] investigated the effects on gait of continuously perturbing the visual support surface in VR and when VR was combined with a mechanical perturbation. They found that visual perturbations applied with VR challenged gait in a way comparable to the coupled VR and mechanical system. The ability to provide disruptions to balance solely with VR could provide a safer and more affordable way to modulate balance during ambulation, for gait rehabilitation.

This study investigates the ability of discrete visual perturbations to gait applied in VR to directly manipulate and challenge a walker's balance control as measured through changes in their center of mass (CoM) and step length (SL). Discrete visual perturbations differ from continuous perturbations in that they are more unpredictable, requiring a larger compensatory response (rather than predictive) that more readily reflects the direct effect of the visual perturbation. During the task, participants were asked to walk on a treadmill as they moved through a virtual environment. As they walked, discrete medial-lateral (left/right) shifts of the visual scene where applied in VR. We hypothesized that participants would: (1) interpret the visual shift of the scene as a challenge to balance control and compensate by shortening their step length while moving the average center of mass (aCoM) excursion in a direction opposite of the visual shift in the scene and (2) exhibit limited visual adaptation to the discrete perturbation with exposure. 


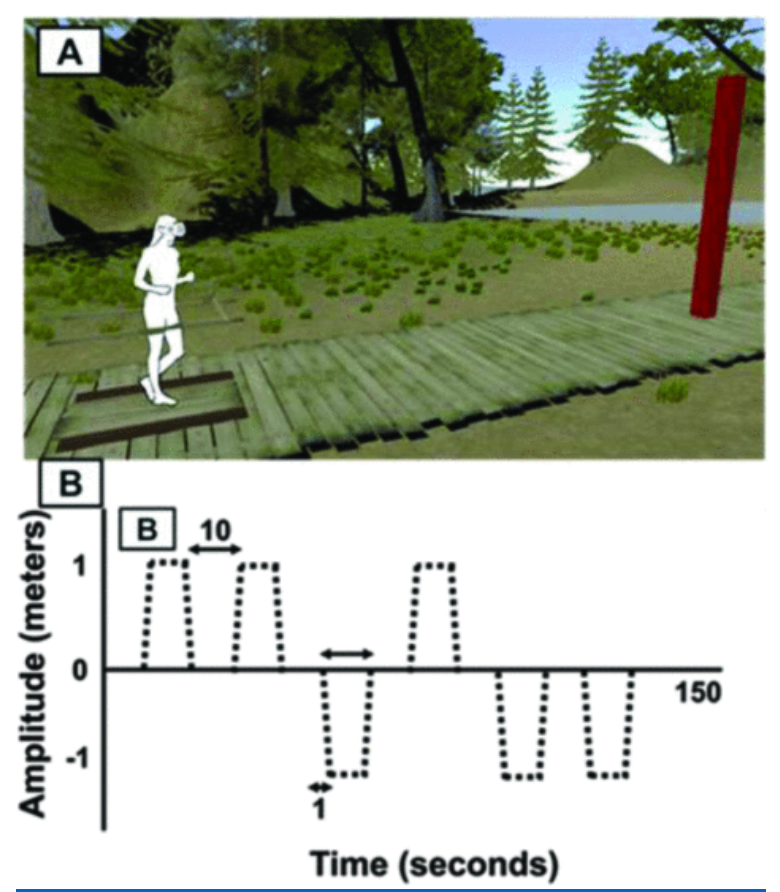

Figure 1. Experimental setup. (A) Virtual scene with the treadmill and red pole. (B) Discrete medial-lateral perturbation applied to the visual scene.

\section{SECTION II. Methods}

\section{A. Experimental Design}

Thirteen healthy young adults participated in the study (mean age: 22.9, standard deviation (SD): 2.3 years; height: 1.75, SD .08 meters; weight: 76.0, SD $15.2 \mathrm{~kg} ; 4$ female and 9 male). Participants had no reported orthopedic or neurological deficiencies, or recent (less than twelve months) lower extremity surgeries. The study was approved by the Institutional Review Board at Marquette University and all participants provided informed written consent prior to participation in the study.

During the experiment, participants walked on a Woodway split belt treadmill for 5 minutes at their selfselected speed. Next, participants donned a Vive head mounted display (HMD) that provided an immersive virtual environment with a $100^{\circ}$ horizontal and $110^{\circ}$ vertical field of view at a resolution of $1080 \times 1200$ pixels per eye and a $60 \mathrm{~Hz}$ refresh rate. Participants walked on the treadmill for an additional 5 minutes to acclimate to the VR system. As they walked, their movement through virtual environment was coupled to the speed of the treadmill. The VR scene consisted of a wooden bridge across a lake with a red pole (8 meters tall) placed 2.5 meters in front of the participant (Figure 1a). Participants were instructed to maintain their head and eye fixation on the pole as they moved through the environment to control for changes in ocular disparity and rotations of the scene due to head movement. The virtual scene was created in Unity (v5.5, Unity Technologies, San Francisco, CA) using the SteamVR plugin (v2.4.5). For safety, the virtual bridge included handrails mapped to the physical handrails of the treadmill $( \pm 1 \mathrm{~cm})$. Participants also wore a safety harness that was connected to both a support frame above the participant and to the treadmill frame in front of the participant to reduce the risk of falls and walking off the treadmill.

During the experiment, participants completed a series of three 2.5-minute trials during which 4-6 discrete mediallateral (left/right) shifts were applied to the virtual environment (Figure 1b). During these discrete visual perturbations (DVP), the participant's viewpoint, the virtual representation of the treadmill, and the pole were moved to the left or the right 1 meter over 1 second relative to the bridge/scene. The position was held for 9 
seconds before returning to the "origin" position in the middle of the bridge over 1 second. Perturbations were presented every 10 seconds with the direction of the shift (left/right) counterbalanced to control for learning and adaptation. Participants completed 6 trials of each condition by the end of the experiment. As participants walked on the treadmill, gait kinematics were measured using a 13-camera Optitrack Flex-13 motion capture system (Natural Point, Corvalis, OR). Kinematic data were measured at $120 \mathrm{~Hz}$ using a 27-marker modified HelenHayes model. Four infrared markers were placed on the upper body (right and left acromioclavicular, C7 and the clavicle). Five markers were placed on the pelvis (right and left anterior superior iliac spine (ASIS), right and left greater trochanter, and the sacrum), and twelve additional markers were placed on the right and left medial knee, lateral knee, medial ankle, lateral ankle, second meta head and fifth meta head. Six rigid clusters, each consisting of three markers organized in an equilateral triangle, were placed on the right and left thigh, shank, and heel.

\section{B. Analysis and Statistical Methods}

Anatomical markers from the motion capture system were labelled in AMASS and biometric processing was completed in Visual3D (C-Motion, Germantown, MD). Subsequent data analysis was performed in MATLAB (2017b, MathWorks, Natick, MA) using in-house scripts. Gait events were identified using the vertical positional data of the heel markers together with the marker trajectory data and checked for consistency with the kinematic model.

The pelvis was modelled continuously using at least three of the five tracking markers and was used to define the medial-lateral center of mass (CoM, Equation 1).

$$
\text { CoM }=\text { SACR }+0.105 *\left[\left(R_{H} P_{-} J C+\text { LHIP_JC }\right) / 2-S A C R\right] ;
$$

where, SACR is the position of the sacrum marker, LHIP_JC and RHIP_JC are the left and right hip joint center positions found using visual3D, and .105 is a constant corresponding to the proximal distance from the hip joint[9]. For each leg, the average center of mass (aCoM) was found over each gait cycle relative to heel strike (HS) to account for normal fluctuations in CoM during walking,

$$
\operatorname{aCoM}=\left(\sum_{t=1}^{N} \operatorname{CoM}_{i}\right) / N
$$

(2)

where $C o M_{i}$ is the center of mass of the $i^{\text {th }}$ time point relative to the previous heel strike, $N$ is the number of data points from the previous heel strike to the current heel strike. Step length was calculated for each leg as the anterior-posterior distance between the lateral ankle markers during heel strike and was normalized to the participant's leg length.

To characterize the effect of the discrete visual perturbations, the straddle step (SS; step during the perturbation) and $1^{\text {st }}-3^{\text {rd }}$ steps (defined at heel strike) after initiation of the visual perturbation (labelled S1, S2, and S3 respectively) were recorded for comparison relative to baseline. Discrete visual perturbations were grouped based on the direction in which the perturbation was applied relative to the participant's viewpoint: from the origin to the right (O2R), from the right back to the origin ( $\mathrm{R} 2 \mathrm{O})$, from the origin to the left (O2L), and from the left back to the origin ( $\mathrm{L2O}$ ). Changes in step length and $\mathrm{aCoM}$ were examined relative to the threestep average before the perturbation was applied. Leg symmetry was assumed, and measurements were averaged between the left and right legs. 
The mean ( \pm 1 standard deviation) changes in step length and aCoM were calculated for each participant and perturbation condition (O2R, R2O, O2L, L2O). A repeated measures analysis of variance (ANOVA) was performed across steps (SS, S1, S2 and S3) for each perturbation condition to test separately for significant changes in step length and aCoM over time. To examine the effect of repeated visual perturbations over time, paired T-tests were used to compare the aCoM on the $2^{\text {nd }}$ step (S2) across trials. All statistical analyses were performed using SPSS (SPSS Inc., Chicago, IL) and MATLAB.

\section{SECTION III. Results}

\section{A. Changes in Gait Caused by Visual Perturbations}

Following a discrete visual perturbation, step length during the second step (S2) (figure 2A) decreased relative to the straddle step (SS), $(p<.05)$ for visual shifts of the scene to the right of the path $(O 2 R, R 2 O)$ and for shifts away from the path on the left (O2L). The change in aCoM was significant across steps for all conditions relative to $\mathrm{S} 2$ (figure $2 \mathrm{~A})$. When the visual scene was shifted to the right $(\mathrm{O} 2 \mathrm{R})$, aCOM excursion was significantly less for the straddle step (SS; $p<0.001)$, first $(S 1 ; p<0.01)$ and third $(S 3 ; p<0.05)$ steps compared to the second step. aCOM excursion for the other perturbation conditions followed a similar trend with $S S(p<0.01), S 1(p<0.01)$ and S3 $(p<0.05)$, significantly less than $\mathrm{S} 2$.

\section{B. Adaptation to Discrete Visual Perturbation}

The difference in aCoM excursion for the second step following the shift in the visual scene is shown for the first and sixth trials in Figure 2B. While there was a general decrease in aCoM excursion with trial repetition, the change was not significant for any perturbation conditions $(p>0.1)$.

\section{SECTION IV. Discussion and Conclusion}

When presented with discrete visual perturbations, participants moved their average CoM (aCOM) to compensate for the visually perceived shift in their body position. This finding supports the hypothesis that visual perturbations alone can elicit a change in CoM and demonstrates their potential use as a tool to challenge balance. The aCoM excursion always occurred in the direction opposite the visual perturbation, and was consistently highest on the $2^{\text {nd }}$ step following the perturbation. For a one-meter medial-lateral movement of the visual scene, the average displacement across participants was approximately 5-6 centimeters. This difference between the perturbation and the amount of the subsequent correction may be due in part to the cue conflict between the shift of the visual scene and participant's proprioceptive and vestibular feedback. A change in CoM of 5-6 cm can push the participants' balance from their base of support [10][11], supporting the use of visual perturbations to challenge balance.

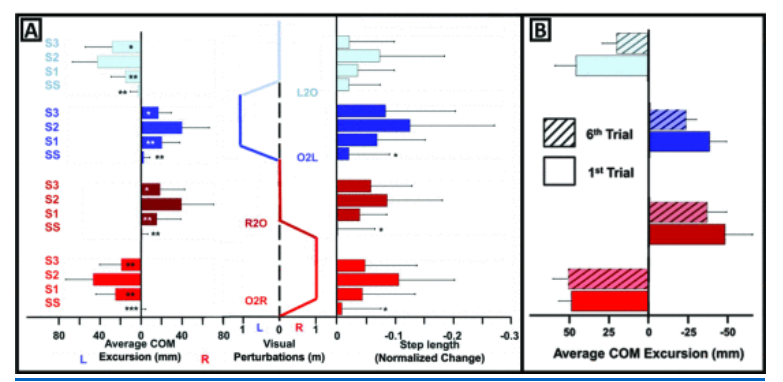

Figure 2. A. Average CoM (aCoM) excursion (left) and normalized change in step length (right) across participants $(n=13)$. Response is shown for the straddle step (SS), and the 1 st-3rd steps after the visual perturbation (S1-S3). Discrete visual perturbations (middle) were applied from the origin to the right $(\mathrm{O} 2 \mathrm{R}, \mathrm{red})$, right back to origin ( $\mathrm{R} 2 \mathrm{O}$, dark red), the origin to the left (O2L, blue), and left to origin (L2O, light blue). B. Comparison of aCoM between the first trial (solid) and sixth trial (stripes). Only the 2 nd step after the 
perturbation ( $\mathrm{S} 2$ ) is shown. No significant difference was found between the 1st and 6th trials. Bars denote group mean and error bars denote one standard deviation. Significant differences are denoted with respect to S2; ${ }^{*}, * *$ and ${ }^{* * *}$ correspond to $p<0.05,0.01$, and 0.001 respectively.

The movement and direction of the response in response to a visual perturbation was consistent with the expected response to a physical perturbation. During postural control, Bronstein et al. found that participants sway in response to discrete visual perturbation [12]. Depending on whether participants fixated on the background or the foreground of the visual display, they swayed either in the same or opposite direction as the visual perturbation respectively. The current results show a similar effect, where since the participants focused here on a pole positioned in front of them, they responded in the opposite direction to the visual stimulus.

While previous studies have reported adaptation over time to visual perturbations during posture [13] and gait [14], our results show that after repeated exposures to discrete visual perturbations, participants did not significantly reduce the change in their aCoM in response to a shift of the visual scene. This difference could be due to the limited number of repetitions applied here or the way in which the visual perturbation was delivered, i.e. as a fast, discrete perturbation, compared to the constant pseudorandom perturbations used in other studies [13]14]. The application of discrete perturbations may be less predictable, eliciting separate distinct corrections that may not be as readily adaptable. Continuous perturbations may cause the participant to adjust using a more predictive strategy[15], while discrete perturbations elicit a more reactive and compensatory strategy[16].

\section{A. Gait and Balance Compensation}

Participants significantly decreased step length in response to discrete shifts of the visual scene in a VR environment. Like aCoM excursion, the largest response came during the second step after the perturbation, followed by a returne to baseline. This change in gait, along with the movement of the aCoM is indicative of a strategy to control balance in response to an external perturbation [17]. The decrease in stride length to control the base of support indicates an effort by participants to compensate for the perceived movement of the CoM in response to the full field movement of the visual scene.

\section{B. Limitations}

Caution is warranted when interpreting the results due to limitations in the experimental design. Motion sickness due to simulated self-movement in the virtual environment could cause increased instability and challenges to gait that may be interpreted as a response to the visual perturbations. The risk of motion sickness was mitigated in this study by allowing participants to take breaks between trials and by maintaining the visual scene presentation at a high framerate. Additionally, participant's responses to the simulator sickness questionnaire indicated that motion sickness was not a significant concern [18]. For this preliminary study, results are reported for a relatively small sample population $(n=13)$. While this can reduce the power to detect differences between experimental conditions, the effects observed here were large relative to the inter-subject variability and were consistent across visual perturbation conditions.

\section{Conclusions}

The presentation of discrete medial-lateral (left/right) shifts of the visual scene in an immersive virtual environment caused significant changes in gait that were sustained across repeated presentations of the visual perturbation. The ability to consistently elicit corrective changes using only changes within a virtual environment could provide a safe and affordable approach for balance and gait rehabilitation. The application of discrete visual perturbations could be used in conjunction with a simple treadmill to encourage adaptation of dynamic balance strategies by shortening stride length while also strengthening the relationship between the base of 
support and movement of the CoM. Additionally, visual perturbations could be used to assess increased reliance on visual feedback for motor planning and balance control, such as associated with age [19]. The use of visual perturbations within a virtual environment to challenge dynamic balance could provide a safer and more affordable avenue for balance rehabilitation by reducing the need for systems that physically perturb balance.

\section{References}

1. M. H. Woollacott and P. F. Tang, "Balance control during walking in the older adult: Research and its implications", Phys. Ther., vol. 77, no. 6, pp. 646-660, 1997.

2. A. Kalron, I. Fonkatz, L. Frid, H. Baransi and A. Achiron, "The effect of balance training on postural control in people with multiple sclerosis using the CAREN virtual reality system: A pilot randomized controlled trial", J. Neuroeng. Rehabil., vol. 13, no. 1, pp. 1-10, 2016.

3. D. Martelli, J. Kang and S. K. Agrawal, "A single session of perturbation-based gait training with the A-TPAD improves dynamic stability in healthy young subjects", IEEE Int. Conf. Rehabil. Robot., pp. 479-484, 2017.

4. D. L. Jaffe, D. A. Brown, C. D. Pierson-Carey, E. L. Buckley and H. L. Lew, "Stepping over obstacles to improve walking in individuals with poststroke hemiplegia", J. Rehabil. Res. Dev., vol. 41, no. 3A, pp. 283-292, 2004.

5. M. J. Casuso-Holgado, R. Martín-Valero, A. F. Carazo, E. M. Medrano-Sánchez, M. D. Cortés-Vega and F. J. Montero-Bancalero, "Effectiveness of virtual reality training for balance and gait rehabilitation in people with multiple sclerosis : a systematic review and meta-analysis", Clin. Rehabiliation, vol. 32, no. 9, pp. 1220-1234, 2018.

6. H. S. Lee, Y. J. Park and S. W. Park, "The Effects of Virtual Reality Training on Function in Chronic Stroke Patients : A Systematic Review and Meta-Analysis", Biomed Res. Int., vol. 2019, pp. 1-12, 2019.

7. R. B. Van Dijsseldonk, L. A. F. De Jong, B. E. Groen, M. V. Hulst, A. C. Geurts and N. L. Keijsers, "Gait Stability Training in a Virtual Environment Improves Gait and Dynamic Balance Capacity in Incomplete Spinal Cord Injury Patients", Front. Neurol., vol. 9, pp. 1-12, 2018.

8. L. Riem, J. Van Dehy, T. Onushko and S. Beardlsey, "Inducing Compensatory Changes in Gait Similar to External Perturbations using an Immersive Head Mounted Display", 2018 IEEE Conf. Virtual Real. 3D User Interfaces, 2018.

9. D. Winter, "Biomechanics and motor control of human movement", 2009.

10. V. Lugade, V. Lin and L. Chou, "Center of mass and base of support interaction during gait", Gait Posture, vol. 33, no. 3, pp. 406-411, 2011.

11. F. A. Y. B. Horak, "Clinical Measurement of Postural Control in Adults", vol. 67, no. 12, 1987.

12. A. Bronstein and D. Buckwell, "Automatic Control of Postural Sway by Visaul Motion Parallax", Exp. Brain Res., vol. 113, no. 9, pp. 243-248, 1997.

13. P. Fransson et al., "Postural instability in an immersive Virtual Reality adapts with repetition and includes directional and gender specific effects", Sci. Rep., vol. 9, no. 1, pp. 1-10, 2019.

14. D. Martelli, B. Xia, A. Prado and S. Agrawal, "Gait adaptations during overground walking and multidirectional oscillations of the visual field in a virtual reality headset", Gait Posture, vol. 67, pp. 251256, 2019.

15. J. D. Thompson and J. R. Franz, "Do kinematic metrics of walking balance adapt to perturbed optical flowf", Hum. Mov. Sci., vol. 54, pp. 34-40, 2017.

16. T. Kajrolkar, F. Yang, Y. C. Pai and T. Bhatt, "Dynamic stability and compensatory stepping responses during anterior gait-slip perturbations in people with chronic hemiparetic stroke", J. Biomech., vol. 47, no. 11, pp. 2751-2758, 2014.

17. L. Hak et al., "Speeding up or slowing downf: Gait adaptations to preserve gait stability in response to balance perturbations", Gait Posture, vol. 36, no. 2, pp. 260-264, 2012.

18. C. Bonnet, E. Fauglorie, M. Riley, B. Bardy and T. Stoffregen, "Motion sickness preceded by unstable displacements of the center of pressure", Hum. Mov. Sci., vol. 25, no. 6, pp. 800-820, 2006. 
19. J. R. Franz, C. A. Francis, M. S. Allen, S. M. O. Connor and D. G. Thelen, "Advanced age brings a greater reliance on visual feedback to maintain balance during walking", Hum. Mov. Sci., vol. 40, pp. 381-392, 2015. 